\title{
Bone resorptive activity in symptomatic and asymptomatic apical lesions of endodontic origin
}

\author{
M. Salinas-Muñoz ${ }^{1}$ M. Garrido-Flores ${ }^{1,2} \cdot$ M. Baeza $^{1,2} \cdot$ P. Huamán-Chipana ${ }^{1}$ \\ J. García-Sesnich ${ }^{1} \cdot$ R. Bologna ${ }^{3} \cdot$ R. Vernal ${ }^{1,4} \cdot$ M. Hernández ${ }^{1,4,5}$ (D)
}

Received: 5 July 2016 / Accepted: 25 January 2017 / Published online: 6 March 2017

(C) The Author(s) 2017. This article is published with open access at Springerlink.com

\begin{abstract}
Objectives The aim of this study is to assess the levels and diagnostic accuracy of a set of bone resorption biomarkers, including TRAP-5, RANKL, and OPG in symptomatic and asymptomatic apical lesions and controls.

Materials and methods Apical tissues from symptomatic and asymptomatic apical periodontitis patients and periodontal ligaments from healthy teeth extracted for orthodontic reasons were processed for tissue homogenization and the levels of TRAP-5, RANKL, and OPG were determined by multiplex assay. Marker levels were analyzed by Kruskal Wallis test, and diagnostic accuracy was analyzed with ROC curves.

Results Higher levels of RANKL, OPG, and RANKL/OPG ratio were determined in both types of apical lesions compared to healthy periodontal ligament, whereas higher TRAP-5 levels were found only in symptomatic apical lesions $(p<0.05)$. OPG, RANKL, and RANKL/OPG ratio showed diagnostic potential to identify apical lesions versus healthy controls (AUC $=0.69, p<0.05$ ); while TRAP-5 showed a potential to discriminate symptomatic versus asymptomatic
\end{abstract}

M. Hernández

mhernandezrios@gmail.com

1 Laboratory of Periodontal Biology, Faculty of Dentistry, Universidad de Chile, Santiago, Chile

2 Department of Conservative Dentistry, Faculty of Dentistry, Universidad de Chile, Santiago, Chile

3 Molecular Pathology Area, School of Dentistry, Universidad de la República UDELAR, Montevideo, Uruguay

4 Dentistry Unit, Faculty of Health Sciences, Universidad Autónoma de Chile, Santiago, Chile

5 Department of Oral Pathology and Medicine, Faculty of Dentistry, Universidad de Chile, Av. Sergio Livingstone 943, Independencia, Santiago, Chile apical periodontitis $(\mathrm{AUC}=0.71, p<0.05)$ and healthy controls $(\mathrm{AUC}=0.83, p<0.05)$.

Conclusions Apical lesions showed higher RANKL and OPG levels than healthy tissues. TRAP-5 levels were the highest in symptomatic apical lesions, suggesting that these represent a progressive state, and showed diagnostic potential.

Clinical relevance Clinically symptomatic apical periodontitis might represent biologically progressive apical lesions based on TRAP5 levels. TRAP5 has diagnostic potential to identify these lesions, representing a candidate prognostic biomarker.

Keywords Symptomatic · Asymptomatic periapical periodontitis $\cdot$ Bone resorption $\cdot$ Biomarkers $\cdot$ TRAP . RANKL $\cdot$ OPG

\section{Introduction}

Apical periodontitis originates from host's immune response to a dominant Gram-negative anaerobic biofilm localized inside the root canal system of the tooth and their respective byproducts. Given the inability to eliminate bacteria, the host attempts to localize the infection and prevent further dissemination at the expense of apical tissue breakdown that results in the formation of an osteolytic apical lesion (AL), the hallmark of chronic forms of apical periodontitis [1].

$\mathrm{AL}$ is heterogeneous from a clinical point of view depending on its association with clinical symptoms, being either symptomatic apical periodontitis (SAP) or asymptomatic apical periodontitis (AAP) [2]. This clinical variability is expected to depend on the dynamic balance between bacterial consortia and the host's response [3-5]. Recent studies support that symptomatic apical periodontitis associates with changes in bacterial load and diversity [4], as well as host's immune 
response, involving interleukin (IL)-1, IL-6, tumor necrosis factor (TNF)- $\alpha$, and matrix metalloproteinase (MMP)-9, respectively [6-8]. Although the clinical diagnosis of symptomatic apical periodontitis is straightforward, its biologic significance remains to be known.

Osteoclasts are the final cellular effectors of bone resorption, determining progression versus healing processes in AL. Osteoclast differentiation and activation from its monocytic precursors is regulated in part through the balance between the receptor activator of nuclear factor $\mathrm{KB}$ (RANK), its ligand (RANKL), and its decoy receptor, osteoprotegerin (OPG). Accordingly, significantly higher RANKL levels have been reported in AL compared with healthy tissues [9]. RANKL/ OPG ratio has also been proposed as an indicator of AL progression $[10,11]$. Tartrate-resistant acid phosphatase (TRAP)5 on the other hand, is an enzyme released along with bone matrix degradation products by active osteoclasts, representing a direct biomarker of osteoclastic activity and bone resorption [12-17].

Higher levels of TRAP-5 are associated with the progression of bone destructive diseases; there is a current need to identify biomarkers for AL progression [18]; however, up to now there are no clinical studies linking TRAP-5 with apical periodontitis $[19,20]$. Furthermore, bone resorptive dynamics in symptomatic and asymptomatic states of apical periodontitis remain unknown [7]. We aimed to assess the levels and diagnostic accuracy of a set of bone resorption biomarkers in AL from patients with clinical diagnoses of SAP, AAP, and healthy periodontal ligaments as controls, including TRAP-5, RANKL, and OPG.

\section{Materials and methods}

\section{Materials}

Tris- $\mathrm{HCl} \mathrm{pH} 7.5, \mathrm{NaCl}, \mathrm{CaCl}_{2}$, and Triton $\mathrm{X}-100$ were purchased from Sigma-Aldrich (St Louis, MO, USA) for homogenization buffer preparation. EDTA-free proteinase inhibitor cocktail was purchased from Roche Diagnostics GmbH (Mannheim, Germany). A Milliplex MAP multiplex assay panel (human cancer/metastasis biomarker magnetic bead) and human bone RANKL single-plex panel was obtained from Millipore, Merck KGaA (Darmstadt, Germany).

\section{Methods}

Patients who consulted at the Clinic of Oral Surgery, School of Dentistry, University of Chile, Santiago, Chile, were enrolled if they had a clinical diagnosis of either SAP or AAP in the presence of an apical lesion detected by apical radiography ( $>2 \mathrm{~mm}$ diameter) caused by dental caries in teeth with a clinical diagnosis of nonvital pulp, according to previously defined criteria [3]. Periodontal ligaments obtained from healthy premolars extracted for orthodontic reasons were used as controls as previously described [21,22]. Exclusion criteria included systemic illness or previous antibiotics or nonsteroidal anti-inflammatory treatment during a 6-months period before the study [23]. All procedures were performed in accordance with the ethical standards of the institutional research and ethics committee and with the Helsinki declaration. The investigation protocol was clearly explained to all the participants of this study. Each participant signed an informed consent or corresponding forms for their legal guardians in case of underage patients after the risks and benefits of participation were described. A total of 52 apical lesions from patients with SAP $(n=17)$ and AAP $(n=35)$, and periodontal ligament samples from healthy volunteers $(n=24)$ were obtained. After tooth extraction, apical lesions and healthy periodontal ligaments were extracted by surgical separation from the tooth surface with curettes and then stored at $-20{ }^{\circ} \mathrm{C}$ until processed for tissue homogenization and multiplex assay.

\section{Tissue homogenates and multiplex assay}

After thawing, tissue samples from AL $(n=52)$ and control periodontal ligaments $(n=24)$ were weighted. Protein extracts were obtained by manual homogenization in $50 \mathrm{mM}$ Tris- $\mathrm{HCl}$ $\mathrm{pH} 7.5,0.2 \mathrm{mM} \mathrm{NaCl}, 5 \mathrm{mM} \mathrm{CaCl}_{2}$, and $0.01 \%$ Triton X-100 (Sigma-Aldrich, St Louis, MO) buffer adding EDTA-free proteinase inhibitor cocktail (Roche Diagnostics GmbH, Mannheim, Germany) in a constant ratio of 10:1 $\mu \mathrm{L}$ of buffer per milligram of weighted tissue; centrifuged at $10,000 \times \mathrm{g}$ for 6 min at $4{ }^{\circ} \mathrm{C}$ and stored at $-80^{\circ} \mathrm{C}$ until further analysis with Milliplex MAP multiplex assays (human cancer/metastasis biomarker magnetic bead and human bone RANKL singleplex panels, Millipore, Merck KGaA, Darmstadt, Germany), according to the manufacturer's instructions. Data was read through a Luminex platform (Magpix, Millipore, St Charles, MO, USA), and analyzed with the MILLIPLEX AnalystR software (ViageneTech, Carlisle, MA, USA).

\section{Statistical analyses}

Comparisons of TRAP-5, RANKL, and OPG levels between SAP and AAP and controls were analyzed with ANOVA or Kruskal Wallis test using STATA V.11 (StataCorp, College Station, TX, USA), according to data distribution. The evaluation of the diagnostic accuracy of the biomarkers was performed through the construction of ROC curves using SPSS19 software (IBM $®$ Company, Armork, NY, USA) by calculating the area under the curve (AUC). The optimal cutoff points to estimate the highest sensitivity and specificity altogether were assessed by Youden's index. A $p$ value $<0.05$ was considered statistically significant. 


\section{Results}

Controls, asymptomatic apical periodontitis and symptomatic apical periodontitis groups had a mean age of 13.7, 50.1 , and 46.5 years and 7, 12, and 11 were women, respectively. No smokers were reported in the controls, whereas there were six smokers in each apical periodontitis group. Regarding biomarker levels (Fig. 1), significantly higher levels of TRAP-5 were observed in SAP in comparison to AAP and healthy controls $(p<0.05)$, and with no differences between the latter two groups. The levels of RANKL, OPG, and the RANKL/OPG ratio were significantly higher in AAP and SAP groups compared with healthy controls $(p<0.05)$, and no differences were found between both apical periodontitis groups.

The diagnostic performance of biomarkers is illustrated with ROC curves (Fig. 2a, b, c) and their respective values are presented in Table 1a, b, c. RANKL, OPG, and RANKL/ OPG demonstrated statistically significant $(p<0.05)$ diagnostic accuracy to identify AAP versus healthy controls, where the highest performance corresponded to RANKL (area under the curve $=0.77,95 \%$ CI $0.65-0.89$ ), [Fig. $2 \mathrm{a}$ and Table 1a ]. Optimal cut-off points were obtained for each marker by using Youden's index. OPG showed the best performance, with a sensitivity of $80 \%$ and a specificity of $69 \%$ at a cut-off point of $50 \mathrm{pg} / \mathrm{mL}$, followed by RANKL and RANKL/OPG ratio.

In the construction of ROC curves for the diagnosis of SAP versus healthy controls [Fig. $2 \mathrm{~b}$ and Table $1 \mathrm{~b}$ ], TRAP$5(\mathrm{AUC}=0.83,95 \%$ CI $0.68-0.98)$, and RANKL $(\mathrm{AUC}=0.83,95 \%$ CI $0.71-0.95)$ were the markers with the highest accuracy, followed by RANKL/OPG ratio and OPG $(p<0.05)$. According to Youden's index, TRAP-5 showed a sensitivity of $71 \%$, a specificity of $100 \%$, at a cut-off point of $12.48 \mathrm{ng} / \mathrm{mL}$, followed by RANKL with a sensitivity of $77 \%$ and a specificity of $69 \%$ at a cut-off point of $90.64 \mathrm{pg} / \mathrm{mL}$.

TRAP-5 (AUC $=0.71,95 \%$ CI $0.54-0.89$ ) was the only biomarker that showed potential to discriminate between SAP and AAP [Fig. 2c) and Table 1c, $p<0.05]$. TRAP-5 showed a sensitivity of $73 \%$ and a specificity of $80 \%$ at a cut-off point of $12.54 \mathrm{ng} / \mathrm{mL}$.

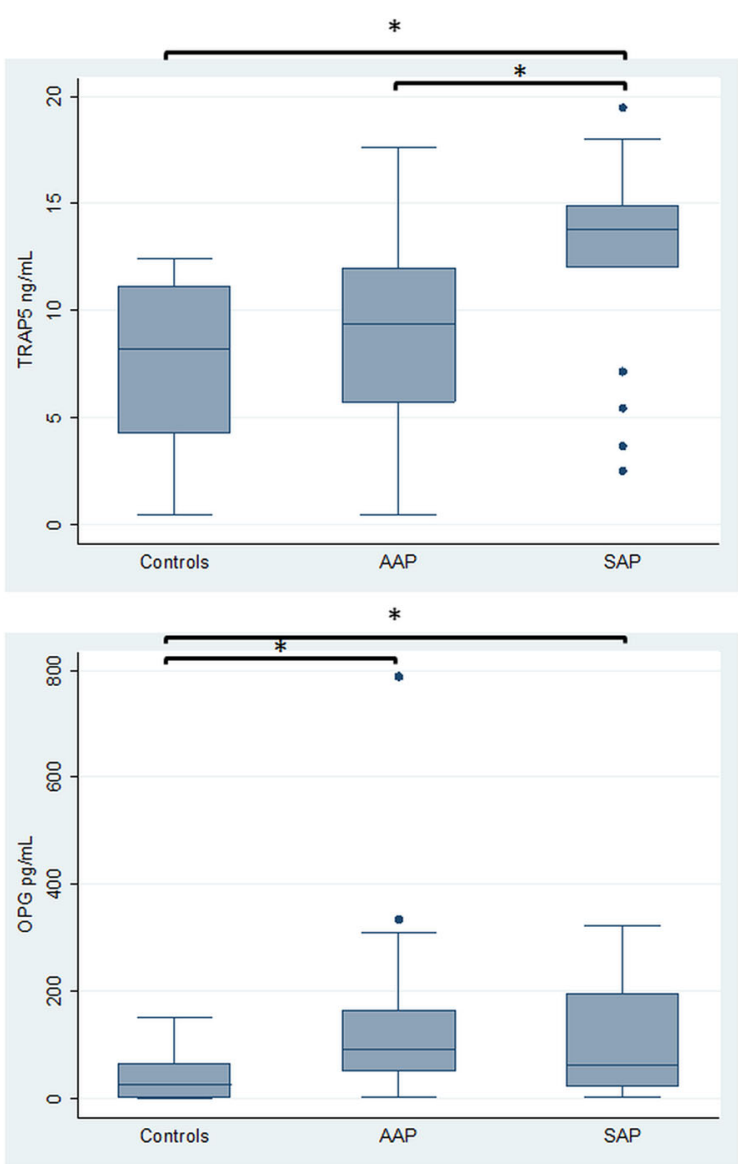

Fig. 1 Bone resorption biomarkers levels in healthy controls, AAP and SAP. Control: healthy periodontal ligaments; $A A P$ asymptomatic apical periodontitis; SAP symptomatic apical periodontitis; TRAP5 tartrateresistant acid phosphatase-5; RANKL receptor activator for nuclear factor

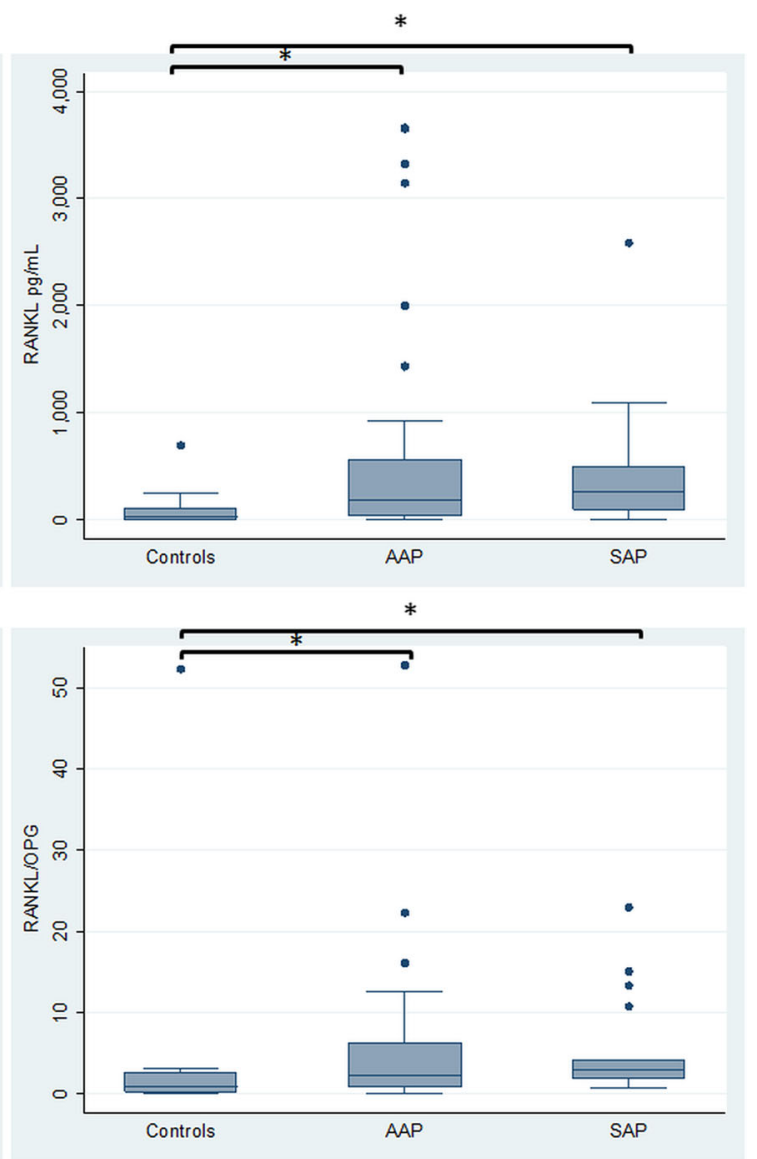

kB ligand; $O P G$ osteoprotegerin; $R A N K L / O P G$ ratio between RANKL and OPG. Bars and asterisks represent significant $(p<0.05)$ pairwise comparisons (ANOVA and Tukey test for TRAP-5 and Kruskal Wallis test for RANKL, OPG, and RANKL/OPG ratio) 

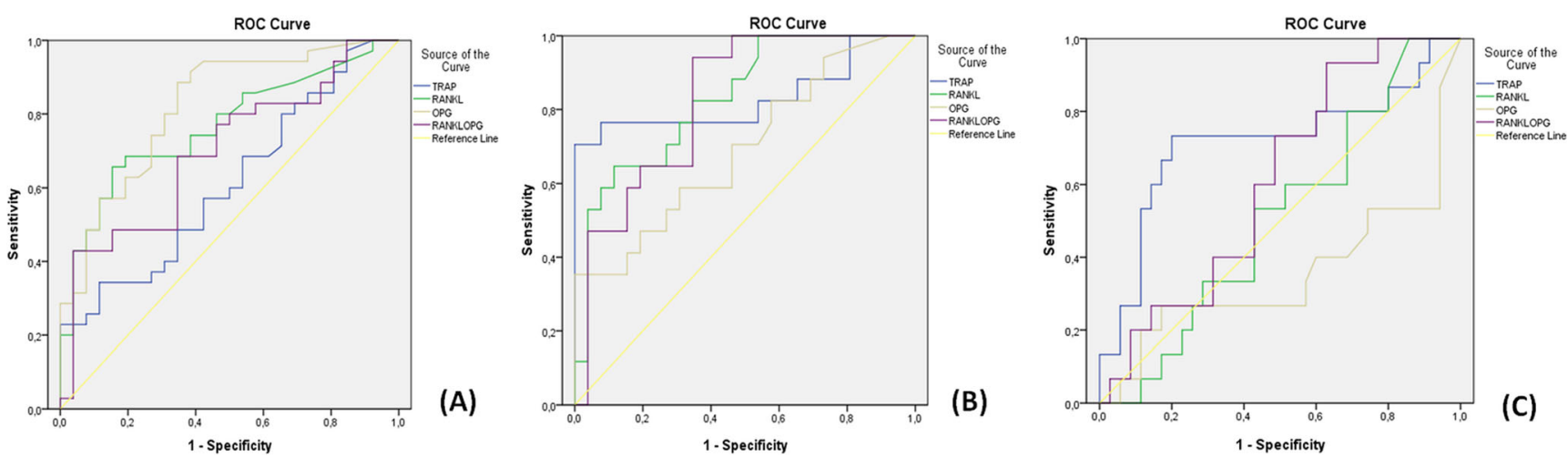

(TRAP), green receptor activator for nuclear factor $\mathrm{kB}$ ligand (RANKL), gray osteoprotegerin (OPG), purple ratio between RANKL, and OPG, yellow reference line

versus controls (b), and AAP versus SAP (c). AAP asymptomatic apic periodontitis; $S A P$ symptomatic apical periodontitis; $y$ axis: sensitivity, $x$ axis: 1-specificity. Lines: blue tartrate-resistant acid phosphatase-5

\section{Discussion}

AL results from an imbalanced osteoclastic activity induced by the immuno-inflammatory response to endodontic bacterial infection [24]. Clinically symptomatic ALs have been suggested to represent an immunologically active stage of the disease [7, 8], but up to now, bone resorptive activity had not been established. In the present study, we assessed the levels of bone resorption markers in symptomatic and asymptomatic AL and healthy periodontal ligament as controls and found statistically significant higher levels of RANKL and OPG in both types of apical lesions compared to healthy periodontal ligament, while higher TRAP-5 levels were found only in symptomatic AL, suggesting that the later represent progressive lesions. TRAP-5 had diagnostic potential for SAP, representing a potentially useful candidate biomarker for apical periodontitis progression.

Bone resorption is a multistep process where RANK/RANKL/OPG pathway and TRAP-5 play key roles. Through binding RANK, RANKL is among the key osteolytic cytokines that promote osteoclast maturation and activation. OPG on the other hand, is a decoy receptor that prevents the coupling of RANK to RANKL [10]. Unlike the RANKL/RANK axis, which might either result or not in osteoclast differentiation depending on the OPG levels, TRAP-5 is an enzyme released along with bone matrix degradation products by active osteoclasts representing a direct biomarker of osteoclastic activity and bone resorption [12-14, 19, 20].
Table 1 Accuracy of biological markers for the diagnosis of AA versus controls (a), SAP versus controls (b) and AAP versus SAP (c)

\begin{tabular}{lllllc}
\hline Biomarker & Cut-off & Sensitivity & Specificity & Accuracy $($ AUC, CI) & $p$ \\
\hline (a) & & & & & \\
TRAP-5 & $8.45 \mathrm{ng} / \mathrm{mL}$ & 0.57 & 0.58 & $0.61(0.47-0.75)$ & 0.143 \\
RANKL & $122.00 \mathrm{pg} / \mathrm{mL}$ & 0.66 & 0.85 & $0.77(0.65-0.89)$ & $<0.0001$ \\
OPG & $50.0 \mathrm{pg} / \mathrm{mL}$ & 0.80 & 0.69 & $0.82(0.71-0.93)$ & $<0.0001$ \\
RANKL/OPG & 1.05 & 0.69 & 0.66 & $0.70(0.57-0.83)$ & 0.009 \\
(b) & & & & \\
TRAP-5 & $12.48 \mathrm{ng} / \mathrm{mL}$ & 0.71 & 1.00 & $0.83(0.68-0.98)$ & $<0.0001$ \\
RANKL & $90.64 \mathrm{pg} / \mathrm{mL}$ & 0.77 & 0.69 & $0.83(0.71-0.95)$ & $<0.0001$ \\
OPG & $51.50 \mathrm{pg} / \mathrm{mL}$ & 0.59 & 0.69 & $0.69(0.53-0.86)$ & 0.035 \\
RANKL/OPG & 0.99 & 0.94 & 0.65 & $0.82(0.70-0.95)$ & $<0.0001$ \\
(c) & & & & $0.71(0.54-0.89)$ & 0.017 \\
TRAP-5 & $12.54 \mathrm{ng} / \mathrm{mL}$ & 0.73 & 0.80 & $0.51(0.34-0.68)$ & 0.92 \\
RANKL & $170.5 \mathrm{pg} / \mathrm{mL}$ & 0.53 & 0.57 & $0.35(0.16-0.54)$ & 0.10 \\
OPG & $70.50 \mathrm{pg} / \mathrm{mL}$ & 0.40 & 0.60 & $0.61(0.45-0.77)$ & 0.22 \\
RANKL/OPG & 2.40 & 0.73 & 0.51 & & \\
\hline
\end{tabular}

$A A P$ asymptomatic apical periodontitis; SAP symptomatic apical periodontitis; $A U C$ area under the curve; $C I$ confidence interval of 95\%; TRAP5 tartrate-resistant acid phosphatase-5; RANKL receptor activator for nuclear factor $\mathrm{kB}$ ligand; $O P G$ osteoprotegerin; $R A N K L / O P G$ ratio between RANKL and OPG

$p<0.05$ 
In the present study the levels of RANKL, OPG and RANKL/OPG ratio were significantly higher in symptomatic and asymptomatic apical lesions versus healthy controls, whereas no significant differences were found between both lesion types. Previous reports associate high levels of RANKL and OPG with apical lesions [10, 17, 25]. In line with our results, a recent report showed no differences in RANKL and OPG levels between asymptomatic and symptomatic apical lesions, whereas a significantly higher number of gramnegative bacteria along with a positive correlation with OPG were found in the later [26]. Whereas the participants showed an homogeneous distribution by gender, an intrinsic methodologic drawback for including healthy periodontal ligament as controls is the age difference among healthy and AP groups, although it represents the closest histophysiologic counterpart for ALs in humans [22]. Accordingly, higher mRNA expression levels were found for RANKL and OPG in apical granulomas versus periodontal ligaments [10]. Recently, RANKL was identified at higher levels in apical exudates from asymptomatic apical periodontitis compared to irreversible pulpitis, whereas OPG levels remained mostly undetectable [27]. So far, there are no previous studies that associate the RANKL/OPG levels with the clinical status in AP. The increased levels of RANKL, OPG, and RANKL/ OPG ratio in ALs suggest that these lesions display an imbalance towards osteoclast differentiation, though it might not necessarily result in higher osteolysis [10, 27]. Based on our results, these markers did not differentiate between symptomatic and asymptomatic apical lesions.

TRAP-5 levels were significantly higher in symptomatic apical lesions compared with asymptomatic lesions and healthy controls, while no differences were found between asymptomatic lesions and controls. TRAP- 5 has been proposed to be the most reliable marker of bone resorptive activity. Clinical studies, as well as animal and in vitro experimental models, demonstrate that elevated levels of TRAP- 5 can be more specifically explained by an increase in the activity of osteoclasts [12-15, 28]. Accordingly, studies conducted in animal models demonstrate that bone resorption rate is significantly higher during the acute stage of apical periodontitis, compared to its chronic phase, where it becomes slower to finally achieve stabilization [29].

Bone resorption and RANKL production by several cell types is synergistically induced by IL-1, IL- 6 , and TNF- $\alpha$. In line with our results, clinical studies report significantly higher levels of these cytokines in apical lesions versus pulp tissue controls and symptomatic versus asymptomatic apical lesions $[7,8,23]$. Despite the RANKL/OPG ratio has been proposed as an indicator of progression or stability of asymptomatic lesions [10], osteolytic cytokines are redundant. TNF- $\alpha$ and IL-1 can also stimulate osteoclast differentiation via RANKL/RANKindependent mechanisms [30,31], implicating that osteoclastogenesis is only partially accounted by the RANKL/RANK axis. Altogether, these antecedents suggest that inflammation enhances osteoclast differentiation in periapical lesions via RANKL/OPG, but only symptomatic lesions might truly represent an active stage, as reflected by concomitantly elevated RANKL/OPG ratio and TRAP-5 levels. Based on previous reports, it is possible that activation pathways other than RANKL might be induced in symptomatic lesions, resulting in exacerbated bone resorption. The detection of RANKL, OPG, and TRAP at lower levels in healthy periodontal ligament on the other hand might reflect bone homeostasis.

The diagnostic accuracy of OPG, RANKL, and RANKL/ OPG ratio showed potential to identify apical lesions versus healthy controls, but failed to discriminate between AAP and SAP. Thus, OPG and RANKL could be useful in the diagnosis of apical periodontitis; however, they do not differentiate between its clinical forms. TRAP- 5 on the other hand proved to have the highest diagnostic accuracy to discriminate SAP from AAP and healthy controls, presenting potential as a marker for progressive lesions. Accordingly, TRAP levels also demonstrated diagnostic precision to identify marginal chronic periodontitis in a recent study [15]. Currently, apical diagnosis is based solely on clinical and radiological parameters, but they fail to predict treatment outcome in the short term. Thus, TRAP-5 screening in a noninvasive approach might have potential as biomarker for progressive AL to aid clinical decisions in endodontic and restorative procedures in oral fluid samples, such as gingival crevicular fluid, saliva, or in apical exudates during endodontic treatment $[15,32]$. In fact, TRAP levels have already shown a good performance to identify chronic periodontitis in gingival crevicular fluid [15]. Future prospective studies are needed to assess its usefulness as a predictive marker.

\section{Conclusions}

Apical lesions showed higher RANKL and OPG levels than healthy tissues and TRAP-5 levels were the highest in symptomatic apical lesions, suggesting that the later represents a progressive disease state that can be identified by TRAP-5.

\section{Compliance with ethical standards}

Conflict of interest The authors declare that they have no conflict of interest.

Funding The work was supported by the National Fund for Scientific and Technologic Development (FONDECYT), Chile \# 1120138 and 1160741.

Ethical approval This article does not contain any studies with animals performed by any of the authors. All procedures performed in studies involving human participants were in accordance with the ethical standards of the institutional and/or national research committee and with the 1964 Helsinki declaration and its later amendments or comparable ethical standards. 
Informed consent Informed consent was obtained from all individual participants included in the study.

Open Access This article is distributed under the terms of the Creative Commons Attribution 4.0 International License (http:// creativecommons.org/licenses/by/4.0/), which permits unrestricted use, distribution, and reproduction in any medium, provided you give appropriate credit to the original author(s) and the source, provide a link to the Creative Commons license, and indicate if changes were made.

\section{References}

1. Hargreaves KM, Goodis HE, Tay FR, Seltzer S (2012) Seltzer and Bender's dental pulp. Quintessence Pub, Hanover Park 501 p

2. AAE (2009) Consensus Conference Recommended Diagnostic Terminology. J Endod 35(12):1634

3. Gutmann JL, Baumgartner JC, Gluskin AH, Hartwell GR, Walton RE (2009) Identify and define all diagnostic terms for periapical/ periradicular health and disease states. J Endod 35:1658-1674

4. Buonavoglia A, Latronico F, Pirani C, Greco MF, Corrente M, Prati C (2013) Symptomatic and asymptomatic apical periodontitis associated with red complex bacteria: clinical and microbiological evaluation. Odontology 101:84-88

5. Hsiao WW, Li KL, Liu Z, Jones C, Fraser-Liggett CM, Fouad AF (2012) Microbial transformation from normal oral microbiota to acute endodontic infections. BMC Genomics 13:345

6. Ahmed GM, El-Baz AA, Hashem AA, Shalaan AK (2013) Expression levels of matrix metalloproteinase-9 and gramnegative bacteria in symptomatic and asymptomatic periapical lesions. J Endod 39:444-448

7. Prso IB, Kocjan W, Simic H, Brumini G, Pezelj-Ribaric S, Borcic J, Ferreri S, Karlovic IM (2007) Tumor necrosis factor-alpha and interleukin 6 in human periapical lesions. Mediat Inflamm 2007:38210

8. Jakovljevic A, Knezevic A, Karalic D, Soldatovic I, Popovic B, Milasin J, Andric M (2015) Pro-inflammatory cytokine levels in human apical periodontitis: correlation with clinical and histological findings. Aust Endod J 41:72-77

9. Vernal R, Dezerega A, Dutzan N, Chaparro A, Leon R, Chandia S, Silva A, Gamonal J (2006) RANKL in human periapical granuloma: possible involvement in periapical bone destruction. Oral Dis 12:283-289

10. Menezes RGTP, Letra A, Bramante CM, Campanelli AP, de Cássia Figueira R, Sogayar MC, Granjeiro JM, Garlet GP (2008) Differential patterns of RANKL/OPG expression in human periapical granulomas: possible association with progressive or stable nature of the lesions. J Endod 34:932-938

11. Araujo-Pires AC, Francisconi CF, Biguetti CC, Cavalla F, Aranha AM, Letra A, Trombone AP, Faveri M, Silva RM, Garlet GP (2014) Simultaneous analysis of T helper subsets (Th1, Th2, Th9, Th17, Th22, Tfh, Tr1 and Tregs) markers expression in periapical lesions reveals multiple cytokine clusters accountable for lesions activity and inactivity status. J Appl Oral Sci 22:336-346

12. Halleen JM (2003) Tartrate-resistant acid phosphatase 5B is a specific and sensitive marker of bone resorption. Anticancer Res 23:1027-1029

13. Halleen JM, Tiitinen SL, Ylipahkala H, Fagerlund KM, Vaananen HK (2006) Tartrate-resistant acid phosphatase 5b (TRACP 5b) as a marker of bone resorption. Clin Lab 52:499-509

14. Janckila AJ, Takahashi K, Sun SZ, Yam LT (2001) Tartrate-resistant acid phosphatase isoform $5 \mathrm{~b}$ as serum marker for osteoclastic activity. Clin Chem 47:74-80

15. Baeza M, Garrido M, Hernandez-Rios P, Dezerega A, Garcia-Sesnich J, Strauss F, Aitken JP, Lesaffre E, Vanbelle S, Gamonal J et al. (2015)
Diagnostic accuracy for apical and chronic periodontitis biomarkers in gingival crevicular fluid: an exploratory study. J Clin Periodontol

16. Basso FG, Soares DG, Pansani TN, Turrioni AP, Scheffel DL, de Souza Costa CA, Hebling J (2015) Effect of LPS treatment on the viability and chemokine synthesis by epithelial cells and gingival fibroblasts. Arch Oral Biol 60:1117-1121

17. da Costa TA, Silva MJB, Alves PM, Chica JEL, Barcelos EZ, Giani MAA, Garlet GP, da Silva JS, Rodrigues Júnior V, Rodrigues DBR et al. (2015) Inflammation Biomarkers of Advanced Disease in Nongingival Tissues of Chronic Periodontitis Patients. Mediat Inflamm 2015

18. Kvist T, van der Sluis L (2015) Report of the first ESE research meeting - 17(th) October 2014, Amsterdam, the Netherlands: the relationship between endodontic infections and their treatment with systemic diseases. Int Endod J 48:913-915

19. Halleen JM, Alatalo SL, Suominen H, Cheng S, Janckila AJ, Vaananen HK (2000) Tartrate-resistant acid phosphatase 5b: a novel serum marker of bone resorption. J Bone Miner Res 15:1337-1345

20. Garnero P (2014) New developments in biological markers of bone metabolism in osteoporosis. Bone 66:46-55

21. Cavalla F, Osorio C, Paredes R, Valenzuela MA, Garcia-Sesnich J, Sorsa T, Tervahartiala T, Hernandez M (2015) Matrix metalloproteinases regulate extracellular levels of SDF-1/CXCL12, IL-6 and VEGF in hydrogen peroxide-stimulated human periodontal ligament fibroblasts. Cytokine 73:114-121

22. Cavalla F, Reyes M, Vernal R, Alvarez C, Paredes R, GarciaSesnich J, Infante M, Farina V, Barron I, Hernandez M (2013) High levels of CXC ligand 12/stromal cell-derived factor 1 in apical lesions of endodontic origin associated with mast cell infiltration. J Endod 39:1234-1239

23. Garrido M, Dezerega A, Bordagaray MJ, Reyes M, Vernal R, Melgar-Rodriguez S, Ciuchi P, Paredes R, Garcia-Sesnich J, Ahumada-Montalva P et al (2015) C-reactive protein expression is up-regulated in apical lesions of endodontic origin in association with interleukin-6. J Endod 41:464-469

24. Nair PN (2004) Pathogenesis of apical periodontitis and the causes of endodontic failures. Crit Rev Oral Biol Med 15:348-381

25. Belibasakis GN, Rechenberg DK, Zehnder M (2013) The receptor activator of NF-kappaB ligand-osteoprotegerin system in pulpal and periapical disease. Int Endod J 46:99-111

26. Carneiro E, Parolin AB, Wichnieski C, Rosa EA, Silva Neto UX, Westphalen VP, Fariniuk LF, Johann AC (2017) Expression levels of the receptor activator of NF- $\mathrm{KB}$ ligand and osteoprotegerin and the number of gram-negative bacteria in symptomatic and asymptomatic periapical lesions. Arch Oral Biol 73:166-171

27. Rechenberg DK, Bostanci N, Zehnder M, Belibasakis GN (2014) Periapical fluid RANKL and IL-8 are differentially regulated in pulpitis and apical periodontitis. Cytokine 69:116-119

28. Rissanen JP, Suominen MI, Peng Z, Halleen JM (2008) Secreted tartrate-resistant acid phosphatase $5 \mathrm{~b}$ is a Marker of osteoclast number in human osteoclast cultures and the rat ovariectomy model. Calcif Tissue Int 82:108-115

29. Stashenko P, Wang CY, Tani-Ishii N, Yu SM (1994) Pathogenesis of induced rat periapical lesions. Oral Surg Oral Med Oral Pathol 78: 494-502

30. Kim JH, Jin HM, Kim K, Song I, Youn BU, Matsuo K, Kim N (2009) The mechanism of osteoclast differentiation induced by IL1. J Immunol 183:1862-1870

31. Kobayashi K, Takahashi N, Jimi E, Udagawa N, Takami M, Kotake S, Nakagawa N, Kinosaki M, Yamaguchi K, Shima N et al (2000) Tumor necrosis factor alpha stimulates osteoclast differentiation by a mechanism independent of the ODF/RANKL-RANK interaction. J Exp Med 191:275-286

32. Mauricio Garrido AD, Castro-Martínez A, Hernández M (2014) Host-dericed biomarkers in gingival crevicular fluid for complementary diagnosis of apical periodontitis. World J Stomatol 3:19-24 